\title{
Novel method of constructing generalized Hoberman sphere mechanisms based on deployment axes
}

(C) The Author(s) 2019. This article is published with open access at link.springer.com and journal.hep.com.cn

\begin{abstract}
This study proposes a method of constructing type II generalized angulated elements (GAEs II) Hoberman sphere mechanisms on the basis of deployment axes that intersect at one point. First, the constraint conditions for inserting $n$ GAEs II into $n$ deployment axes to form a loop are given. The angle constraint conditions of the deployment axes are obtained through a series of linear equations. Second, the connection conditions of two GAEs II loops that share a common deployable center are discussed. Third, a flowchart of constructing the generalized Hoberman sphere mechanism on the basis of deployment axes is provided. Finally, four generalized Hoberman sphere mechanisms based on a fully enclosed regular hexahedron, arithmetic sequence axes, orthonormal arithmetic sequence axes, and spiral-like axes are constructed in accordance with the given arrangement of deployment axes that satisfy the constraint conditions to verify the feasibility of the proposed method.
\end{abstract}

Keywords deployable mechanism, type II generalized angulated elements, Hoberman sphere mechanism, deployment axes, constraint conditions

\section{Introduction}

Deployable mechanisms are widely used in daily life and aerospace fields, such as deployable tents, roofs, antennas, and solar panels, because of their excellent deployable and stretching properties. Piñero [1] utilized scissor-hinge

Received May 12, 2019; accepted August 18, 2019

Xuemin SUN, Yan-An YAO ( $₫)$

School of Mechanical, Electronic and Control Engineering, Beijing Jiaotong University, Beijing 100044, China

E-mail: yayao@bjtu.edu.cn

Ruiming LI (

Robotics Institute, Beihang University, Beijing 100191, China

E-mail: ruimingli@buaa.edu.cn structures to construct building roofs and designed a deployable mobile theater. Zeigler [2] proposed a selfstabilizing roof based on the shear fork mechanism; the roof increased the structural stability of the studied theater. Escrig and Valcarcel $[3,4]$ obtained various combinations of scissor-like deployable mechanisms through an in-depth analysis of geometry and motion characteristics and constructed complex assemblies with deployable characteristics in one, two, and three dimensions. You [5] proposed an intermediate element that consists of two ordinary scissor-hinge elements and constructed deployable structures with rotational symmetry.

On the basis of scissor-hinge structures, Hoberman [6,7] converted the straight rods of such structures into a pair of angulated elements (AEs) and constructed a series of plane and space (Hoberman sphere) deployable mechanisms. You and Pellegrino [8] analyzed the construction constraint conditions of AEs and proposed two generalized AEs, namely, type I generalized AEs (GAEs I) and type II generalized AEs (GAEs II). Patel and Ananthasuresh [9] described the motion trajectory of a radial motion mechanism by solving the kinematics of the planarcoupled prismatic-revolute-revolute-prismatic (PRRP) mechanism and provided the trajectories of the intermediate hinge points of AEs, GAEs I, and GAEs II. Cai et al. $[10,11]$ analyzed the kinematics and mobility of AEs and angulated scissor-like elements by referring to screw theory. Chen et al. [12] presented an integral mechanism mode for symmetric structures and proposed its kinematics on the basis of symmetric deployable scissor-hinge structures.

Bai et al. [13] introduced angulated-straight elements and the synthesis methods of deployable mechanisms for regular polygons and regular polyhedra. Wohlhart [14,15] proposed deployable mechanisms based on cyclic polyhedra. Wei and Dai [16] used the reciprocating motion of the PRRP chain to arrange a pentahedron and a cube and presented four over-constrained mechanisms with radially reciprocating motion. Li et al. $[17,18]$ proposed reconfigurable, double, and multiple AEs and constructed 
reconfigurable deployable polyhedral mechanisms on the basis of the Platonic polyhedron and semiregular and Johnson polyhedra. Li et al. [19] developed a pair of straight elements degenerated from a pair of AEs and constructed reconfigurable deployable polyhedron mechanisms. Kiper et al. [20] synthesized polyhedral linkages by taking advantage of the Cardan motion along the axes. Shieh [21] presented deployable mechanisms based on a planar four-bar linkage. Huang et al. [22,23] proposed deployable mechanisms derived from the threefold-symmetric deployable Bricard mechanism and irregularly shaped triangular prismoid units. Qi et al. [24] proposed a class of large deployable mechanisms based on plane-symmetric Bricard linkages. Wang and Kong [25] introduced deployable polyhedron mechanisms constructed by connecting spatial single-loop linkages. St-Onge and Gosselin [26] proposed a new design of deployable one-degree-of-freedom mechanisms based on rigid links.

The deployable mechanisms in these studies were constructed by inserting modular linkages into the vertices, edges, and faces of the given polyhedra, and the centers of the polyhedra were normally the deployable centers. However, the number of polyhedra is limited. Hence, we need to explore a highly general method of constructing deployable mechanisms, including regular and irregular ones. Furthermore, irregular deployable mechanisms can provide high application diversity. All deployable mechanisms are deployed along the axes passing the deployable center, which are referred to as deployment axes in this study. As shown in Fig. 1, the angles between the adjacent deployment axes of a regular hexahedron satisfy $\theta_{1}=$ $\theta_{2}=\cdots=\theta_{n}$. Semiregular polyhedra, such as a triangular prism and truncated octahedron, have symmetrical deployment axes. The construction conditions for $n$ arbitrary deployment axes require further investigation.

In this work, we build a cluster of deployment axes that intersect at a common center, analyze the constraint conditions for inserting AEs into three or more arbitrary deployment axes, and propose a novel approach to construct generalized Hoberman sphere mechanisms based on AEs and deployment axes.

The rest of the paper is organized as follows. Section 2 presents the constraint conditions for inserting a GAEs II loop into $n$ deployment axes. Section 3 introduces the construction of Hoberman sphere mechanisms on the basis of deployment axes and the parameters of two adjacent GAEs II loops. Section 4 shows the construction of several Hoberman sphere mechanisms on the basis of given axis arrangements. Section 5 provides the conclusions of this study.

\section{GAEs II loop with $\boldsymbol{n}$ deployment axes}

The AEs proposed by Hoberman [6,7] is shown in Fig. 2(a). The link lengths and angles of the AEs satisfy $A C=B C, D C=E C, \angle A C D=\angle B C E=\pi-\angle B O D$. When points $A$ and $B$ and points $D$ and $E$ move along the lines $A B$ and $D E$, respectively, the trajectory of point $C$ is the line $O C$ passing through point $O$. As shown in Fig. 2(b), the link lengths and angles of GAEs II [8] satisfy $B C / A C=$

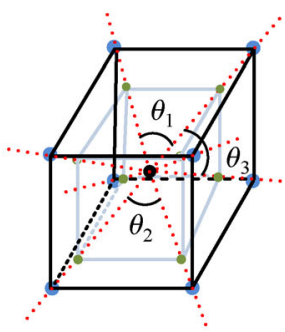

(a)

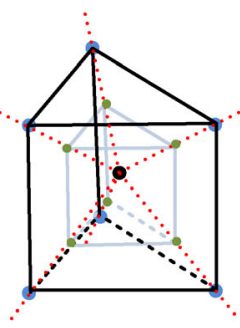

(b)

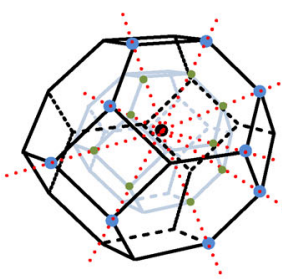

(c)

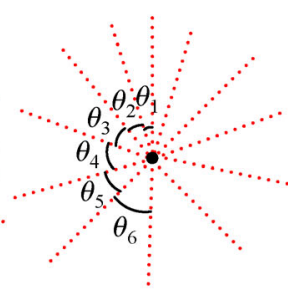

(d)

Fig. 1 Polyhedra and deployment axes. (a) Regular hexahedron; (b) uniform triangular prism; (c) truncated octahedron; (d) arithmetic sequence axes.

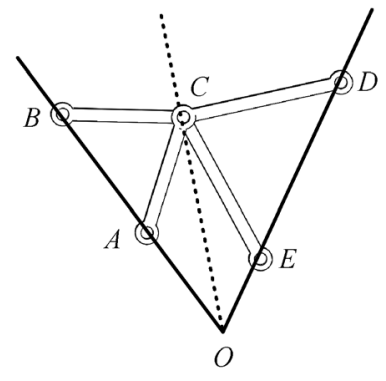

(a)

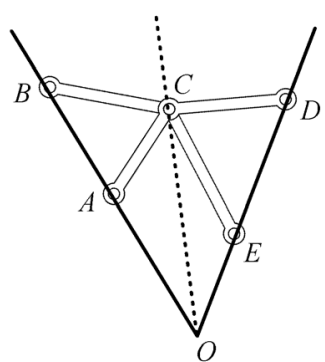

(b)

Fig. 2 Angulated elements and generalized angulated elements. (a) AEs; (b) GAEs II. 
$E C / D C=m$, and $\angle A C D=\angle B C E=\pi-\angle B O D$. GAEs II is similar to AEs; when points $A$ and $B$ and points $D$ and $E$ move along the lines $A B$ and $D E$, respectively, the trajectory of point $C$ is the line $O C$ passing through point $O$. AEs and GAEs II are deployed along lines $O B$ and $O D$, which are the deployment axes, and AEs is a special case of GAEs II when $m=1$. We discuss the construction of highly general Hoberman sphere mechanisms by using GAEs II and the deployment axes.

2.1 Constraint conditions of two GAEs II with three deployment axes

As shown in Fig. 3, two GAEs II (GAEs II-L and GAEs II$\mathrm{R})$ are connected. The constraint conditions of GAEs II-L are $\angle C O F=\theta_{1}, \angle A B F=\angle C B E=\pi-\theta_{1}, B F=l_{1}, A B=l_{2}$, $B E=m_{1} l_{1}, B C=m_{1} l_{2}$, and the constraint conditions of GAEs II-R are $\angle C O H=\theta_{2}, \angle A D H=\angle C D G=\pi-\theta_{2}, A D$ $=l_{3}, D H=l_{4}, D C=m_{2} l_{3}, D G=m_{2} l_{4}$. All of these conditions should be satisfied. $B N$ is perpendicular to $O C$ and intersects $O C$ at point $N . D N^{\prime}$ is perpendicular to $O C$ and intersects $O C$ at point $N^{\prime} . \angle B O F=\theta_{1}^{\prime}, \angle B O C=\theta_{1}^{\prime \prime}$, $\angle C O D=\theta_{2}^{\prime}$, and $\angle H O D=\theta_{2}^{\prime \prime}$.

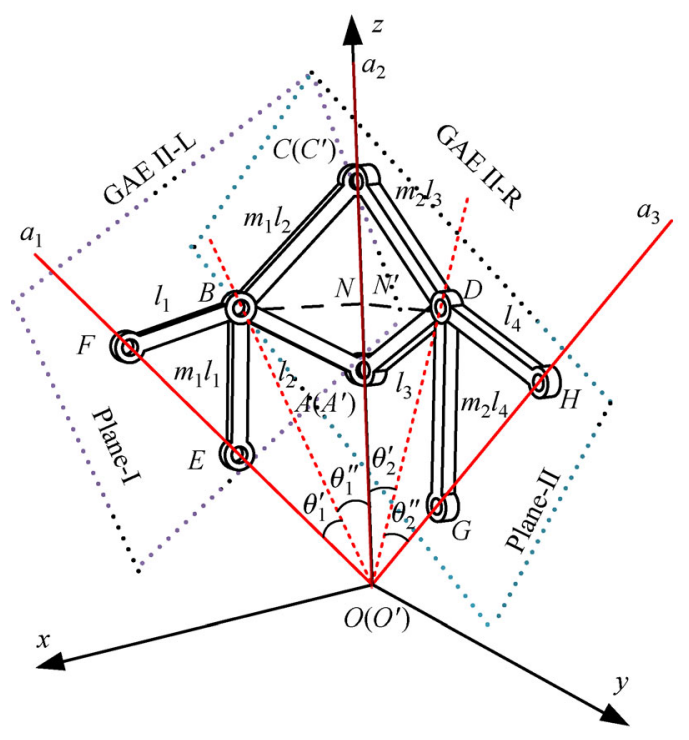

Fig. 3 Cartesian coordinate system of two GAEs II.

Three deployment axes $\left(a_{1}, a_{2}\right.$, and $\left.a_{3}\right)$ are shown in Fig. 3. Deployment axes $a_{1}$ and $a_{2}$ are on plane-I, $a_{2}$ and $a_{3}$ are on plane-II, and $a_{2}$ is the intersection axis of the two planes. A Cartesian coordinate system is established. The origin is set at point $O\left(O^{\prime}\right)$, that is, the intersection of the three deployment axes. The $z$-axis is set along deployment axis $a_{2}$, and the $y$-axis is set along the line that is perpendicular to the $z$-axis and passes through point $O$ on plane-II. According to the right-hand rule, the $x$-axis is perpendicular to the $y O z$ plane. When points $A$ and $C$ and points $E$ and $F$ move along axes $O C$ and $O F$, respectively, the trajectory of point $B$ is line $O B$. The coordinates of point $A$ are $(0,0, a)$, the coordinates of point $C$ are $(0,0, c)$, $\angle B A C=\varphi$, and $\angle D A C=\phi$, where $a, c, \varphi$, and $\phi$ are variables. The coordinates of point $B$ are $\left(b_{x}, b_{y}, b_{z}\right)$, $\angle B O C$ is constant, and the dihedral angle of plane-I and plane-II is set as $\omega$. We can obtain the trajectory of point $B$ as follows:

$$
\left\{\begin{array}{l}
b_{\mathrm{z}} \tan \theta_{1}^{\prime \prime} \cos \omega=b_{y}, \\
b_{\mathrm{z}} \tan \theta_{1}^{\prime \prime} \sin \omega=b_{x} .
\end{array}\right.
$$

The coordinates of point $B$ are $\left(b_{\mathrm{z}} \tan \theta_{1}^{\prime \prime} \sin \omega=b_{x}\right.$, $\left.b_{\mathrm{z}} \tan \theta_{1}^{\prime \prime} \cos \omega=b_{y}, b_{z}\right)$. Given that $A B=l_{2}$ and $B C=m_{1} l_{2}$, we have

$$
\left\{\begin{array}{l}
\left(b_{z} \tan \theta_{1}^{\prime \prime} \sin \omega\right)^{2}+\left(b_{z} \tan \theta_{1}^{\prime \prime} \cos \omega\right)^{2}+\left(b_{z}-c\right)^{2}=m_{1}^{2} l_{2}^{2}, \\
\left(b_{z} \tan \theta_{1}^{\prime \prime} \sin \omega\right)^{2}+\left(b_{z} \tan \theta_{1}^{\prime \prime} \cos \omega\right)^{2}+\left(b_{z}-a\right)^{2}=l_{2}^{2} .
\end{array}\right.
$$

The equation of coordinate $b_{z}$ can be obtained from Eq. (2):

$$
\begin{aligned}
(1 & \left.+\tan ^{2} \theta_{1}^{\prime \prime}\right)\left(1-m_{1}^{2}\right) b_{z}^{2}-2 b_{z}\left(c-m_{1}^{2} a\right)+\left(c^{2}-m_{1}^{2} a^{2}\right) \\
& =0 .
\end{aligned}
$$

Considering that point $B$ is the intersection of the two spheres of Eq. (2) and lies on plane-I, Eq. (3) has one solution or two equal solutions.

Similarly, we let the coordinates of point $D$ be $\left(d_{x}, d_{y}\right.$, $d_{z}$ ). We can obtain the trajectory of point $D$ as

$$
\left\{\begin{array}{l}
d_{z} \tan \theta_{2}^{\prime}=d_{y}, \\
0=d_{x} .
\end{array}\right.
$$

Thus, the equation of coordinate $d_{z}$ is

$$
\begin{aligned}
& \left(1+\tan ^{2} \theta_{2}^{\prime}\right)\left(1-m_{2}^{2}\right) d_{z}^{2}-2 d_{z}\left(c-m_{2}^{2} a\right)+\left(c^{2}-m_{2}^{2} a^{2}\right) \\
& \quad=0 .
\end{aligned}
$$

Given that point $D$ is the intersection of the two spheres and lies on plane-II, Eq. (5) has one solution or two equal solutions.

From the analysis of Eqs. (3) and (5), we can derive the following cases.

Case 1. If $m_{1}=m_{2}=1$ and Eqs. (3) and (5) have one solution, we have

$$
b_{y}=\frac{a+c}{2}=d_{y}
$$

Hence, $B D$ is perpendicular to $C A$ and bisects $C A$.

$$
\begin{aligned}
& B N=O N \tan \theta_{1}^{\prime \prime}, \\
& D N=O N \tan \theta_{2}^{\prime} .
\end{aligned}
$$


In the right triangles $A B N$ and $A N D$, we have

$$
\begin{aligned}
& l_{2}^{2}=B N^{2}+C N^{2}, \\
& l_{3}^{2}=D N^{2}+C N^{2} .
\end{aligned}
$$

Hence,

$$
l_{2}^{2}-l_{3}^{2}=B N^{2}-D N^{2}=O N^{2}\left(\tan ^{2} \theta_{1}^{\prime \prime}-\tan ^{2} \theta_{2}^{\prime}\right),
$$

where $\tan ^{2} \theta_{1}^{\prime \prime}-\tan ^{2} \theta_{2}^{\prime}$ is quantitative because the left side of Eq. (11) is quantitative and $O N^{2}$ on the right side is a variable. To ensure that the equation is always true, we let $l_{2}^{2}-l_{3}^{2}=0$ and $\tan ^{2} \theta_{1}^{\prime \prime}-\tan ^{2} \theta_{2}^{\prime}=0$. We obtain

$$
\begin{gathered}
\theta_{1}^{\prime \prime}=\theta_{2}^{\prime}, \\
l_{2}^{2}=l_{3}^{2} .
\end{gathered}
$$

Therefore, the two GAEs II inserted into the three deployment axes should satisfy the constraint conditions:

$$
\left\{\begin{array}{l}
l_{2}=l_{3}, \\
\theta_{1}^{\prime \prime}=\theta_{2}^{\prime} .
\end{array}\right.
$$

Case 2. If $m_{1} \neq 1, m_{2} \neq 1$, the discriminants $\Delta_{B}$ and $\Delta_{D}$ of Eqs. (3) and (5) are equal to 0, and Eqs. (3) and (5) have two equal solutions, then we derive

$$
\frac{\tan ^{2} \theta_{1}^{\prime \prime}}{\tan ^{2} \theta_{2}^{\prime}}=\frac{m_{1}^{2}\left(m_{2}^{2}-1\right)\left(c^{2}-m_{2}^{2} a^{2}\right)}{m_{2}^{2}\left(m_{1}^{2}-1\right)\left(c^{2}-m_{1}^{2} a^{2}\right)} .
$$

Given that $\theta_{1}^{\prime \prime}, \theta_{2}^{\prime}, m_{1}$, and $m_{2}$ are quantitative, $a$ and $c$ are variables. We let $T=\frac{\tan ^{2} \theta_{1}^{\prime \prime}}{\tan ^{2} \theta_{2}^{\prime}} \frac{m_{2}^{2}\left(m_{1}^{2}-1\right)}{m_{1}^{2}\left(m_{2}^{2}-1\right)}$ and obtain the following from Eq. (15):

$$
T=\frac{c^{2}-m_{2}^{2} a^{2}}{c^{2}-m_{1}^{2} a^{2}} \Rightarrow(T-1) a^{2}=\left(T m_{1}^{2}-m_{2}^{2}\right) c^{2},
$$

where $a^{2} / c^{2}$ is not quantitative, so

$$
\left\{\begin{array} { l } 
{ T - 1 = 0 , } \\
{ T m _ { 1 } ^ { 2 } - m _ { 2 } ^ { 2 } = 0 . }
\end{array} \Rightarrow \left\{\begin{array}{l}
T=1, \\
m_{1}=m_{2} .
\end{array}\right.\right.
$$

Thus,

$$
T=\frac{\tan ^{2} \theta_{1}^{\prime \prime}}{\tan ^{2} \theta_{2}^{\prime}} \frac{m_{2}^{2}\left(m_{1}^{2}-1\right)}{m_{1}^{2}\left(m_{2}^{2}-1\right)}=\frac{\tan ^{2} \theta_{1}^{\prime \prime}}{\tan ^{2} \theta_{2}^{\prime}}=1 .
$$

We have

$$
\theta_{1}^{\prime \prime}=\theta_{2}^{\prime} .
$$

Substituting Eq. (19) into Eqs. (3) and (5) yields

$$
b_{z}=d_{z} \text {. }
$$

We obtain

$$
l_{2}=l_{3} \text {. }
$$

Therefore, the two GAEs II inserted into the three deployment axes should satisfy the constraint conditions

$$
\left\{\begin{array}{l}
l_{2}=l_{3}, \\
\theta_{1}^{\prime \prime}=\theta_{2}^{\prime}, \\
m_{1}=m_{2} .
\end{array}\right.
$$

Case 3. If $m_{1}=1, m_{2} \neq 1$, the discriminant $\Delta_{D}$ of Eq. (5) is equal to 0, Eq. (3) has one solution, and Eq. (5) has two equal solutions, then the two GAEs II cannot satisfy the constraint conditions for being inserted into the deployment axes at this time.

In summary, the constraint conditions of the two GAEs II inserted into the three deployment axes are presented in Eq. (22), and the length of $O B$ (distance from the origin to point $B$ ) is always equal to the length of $O D$ (distance from the origin to point $D$ ).

\subsection{Constraint conditions of $n$ GAEs II with $n$ deployment} axes

According to the constraint conditions of two GAEs II with three deployment axes, the following $n$ GAEs II are inserted into $n$ deployment axes, and these deployment axes intersect at one point and form a loop. As shown in Fig. 4, according to Eq. (22), the constraint conditions can be summarized as follows:

$$
\left\{\begin{array}{l}
l_{2 i-2}=l_{2 i-1}, \\
l_{2 n}=l_{1}, \\
m_{i-1}=m_{i}, \\
\theta_{i-1}^{\prime \prime}=\theta_{i}^{\prime}, \\
\theta_{n}^{\prime \prime}=\theta_{1}^{\prime},
\end{array}\right.
$$

where $l_{2 i-1}$ and $l_{2 i}$ are the lengths of the links of the $i$ th

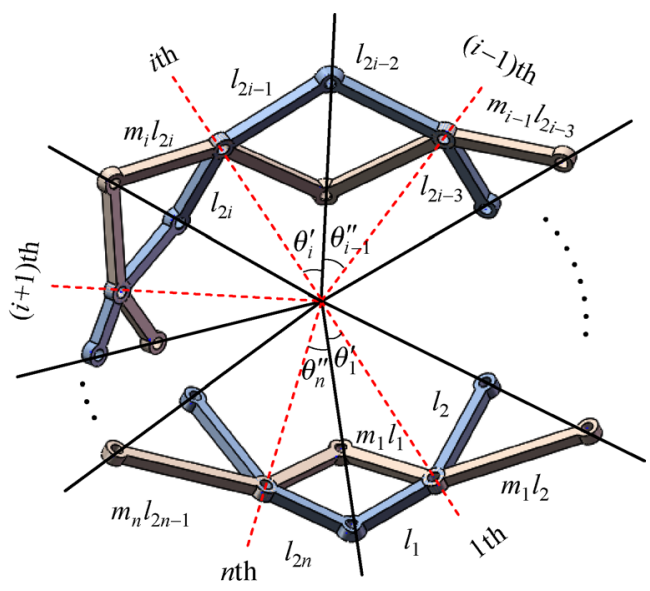

Fig. 4 Loop of $n$ GAEs II. 
GAEs II, $m_{i}$ is the ratio of the $i$ th GAEs II, $\theta_{i}^{\prime}$ and $\theta_{i}^{\prime \prime}$ are the angles of the deployment axes of the $i$ th GAEs II, and $i$ $=2,3, \ldots, n$.

\subsection{Angle constraint conditions of the deployment axes}

As shown in Fig. 5, GAEs II satisfies the constraint conditions $\alpha=\beta=\pi-\theta_{1}, B F=l_{1}, A B=l_{2}, B E=m_{1} l_{1}$, and $B C=m_{1} l_{2}$, where $m_{1}$ is a positive number. In the triangles $O B F$ and $O B A$, the following equation is obtained with the sine theorem.

$$
\frac{l_{1}}{\sin \theta_{1}^{\prime}}=\frac{l_{2}}{\sin \theta_{1}^{\prime \prime}} .
$$

Therefore, for a loop, the lengths of the links of GAEs II can be obtained from Eqs. (23) and (24) as long as the angles are given.

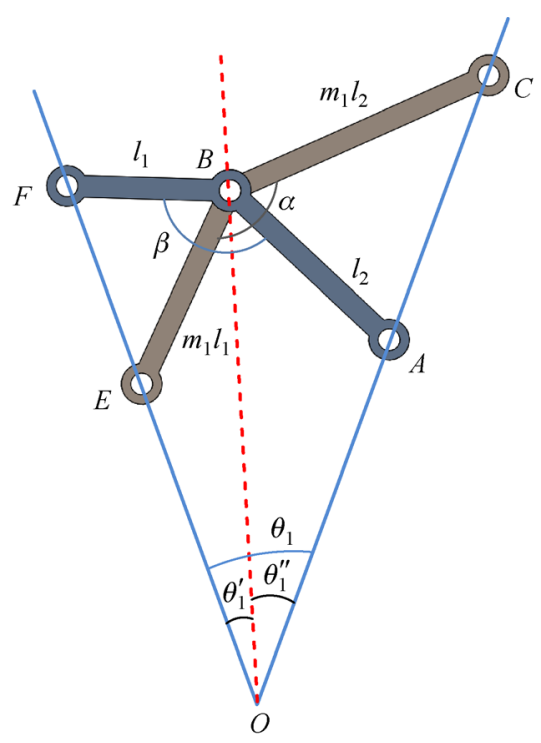

Fig. 5 Parameters of GAEs II.
Naturally, the angles of the $n$ deployment axes are $\theta_{1}$, $\theta_{2}, \ldots, \theta_{n}$ and satisfy

$$
\left\{\begin{array}{l}
\theta_{1}^{\prime}+\theta_{1}^{\prime \prime}=\theta_{1}, \\
\theta_{2}^{\prime}+\theta_{2}^{\prime \prime}=\theta_{2}, \\
\quad \cdots \\
\theta_{n-1}^{\prime}+\theta_{n-1}^{\prime \prime}=\theta_{n-1}, \\
\theta_{n}^{\prime}+\theta_{n}^{\prime \prime}=\theta_{n} .
\end{array}\right.
$$

According to Eq. (23), a loop based on the deployment axes should meet the constraint conditions $\theta_{n}^{\prime \prime}=\theta_{1}^{\prime}$, $\theta_{1}^{\prime \prime}=\theta_{2}^{\prime}, \ldots, \theta_{n-1}^{\prime \prime}=\theta_{n}^{\prime}$, which are respectively expressed as $x_{1}, x_{2}, \ldots, x_{n}$. Then, the following equations can be obtained:

$$
\left\{\begin{array}{c}
x_{1}+x_{2}=\theta_{1}, \\
x_{2}+x_{3}=\theta_{2}, \\
x_{3}+x_{4}=\theta_{3}, \\
x_{4}+x_{5}=\theta_{4}, \\
\cdots \\
x_{n}+x_{1}=\theta_{n} .
\end{array}\right.
$$

The augmented matrix of Eq. (26) is

$$
\boldsymbol{B}=\left(\begin{array}{cccccc|c}
1 & 1 & 0 & \ldots & 0 & 0 & \theta_{1} \\
0 & 1 & 1 & \ldots & 0 & 0 & \theta_{2} \\
. & . & . & & . & . & . \\
. & . & . & & . & . & . \\
. & . & . & & . & . & . \\
0 & 0 & 0 & \ldots & 1 & 1 & \theta_{n-1} \\
1 & 0 & 0 & \ldots & 0 & 1 & \theta_{n}
\end{array}\right) .
$$

Elementary row transformations are performed on the augmented matrix of linear equations.

When $n$ is even, we obtain

$$
\boldsymbol{B}=\left(\begin{array}{cccccc|c}
1 & 1 & 0 & \ldots & 0 & 0 & \theta_{1} \\
0 & 1 & 1 & \ldots & 0 & 0 & \theta_{2} \\
. & . & . & & . & . & . \\
. & . & . & & . & . & . \\
. & . & . & & . & . & . \\
0 & 0 & 0 & \ldots & 1 & 1 & \theta_{n-1} \\
1 & 0 & 0 & \ldots & 0 & 1 & \theta_{n}
\end{array}\right) \rightarrow \ldots \rightarrow\left(\begin{array}{cccccc|c}
1 & 1 & 0 & \ldots & 0 & 0 & \theta_{1} \\
0 & 1 & 1 & \ldots & 0 & 0 & \theta_{2} \\
. & . & . & & . & . & . \\
. & . & . & & . & . & . \\
. & . & . & & . & . & \theta_{n-1} \\
0 & 0 & 0 & \ldots & 1 & 1 & 0 \\
0 & 0 & 0 & \ldots & 0 & 0 & \theta_{n}-\theta_{1}-\cdots-\theta_{n-1}+\theta_{2}+\cdots+\theta_{n-2}
\end{array}\right) .
$$

When $n$ is odd, we obtain 


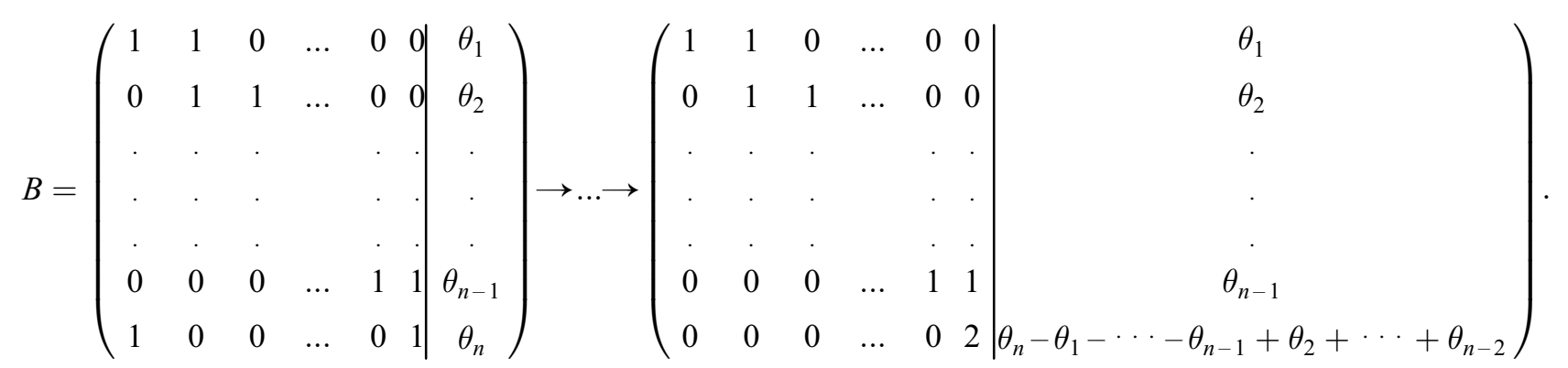

In conclusion, when $n$ is even, $\theta_{1}+\theta_{3}+\cdots+\theta_{n-1}=$ $\theta_{n}+\theta_{2}+\cdots+\theta_{n-2}$ is a sufficient and necessary condition for the existence of a solution, and infinite solutions exist at this time. When $n$ is odd, the equation has a unique solution. However, when the loop is constructed, the angles must all be positive. Thus, a loop can be constructed only when Eq. (26) has a positive solution. In addition, the angles of any single loop must satisfy Eq. (26).

\section{Construction of a Hoberman sphere mechanism based on deployment axes and GAEs II loops}

To insert multiple GAEs II into multiple loops based on deployment axes, we must introduce platforms for the connections among adjacent loops. The lengths of the platforms have been studied, but the constraint conditions of the connections among loops still need to be discussed.

We consider the connection conditions of two loops. As shown in Fig. 6(a), two GAEs II loops (loops I and II) have a common deployable center, which is also the intersecting point of deployment axes. The two GAEs II loops can be simplified as in Fig. 6(b). The term $r$ th is the common GAEs II of loops I and II. Thus, according to Eqs. (22) and (23), we can derive

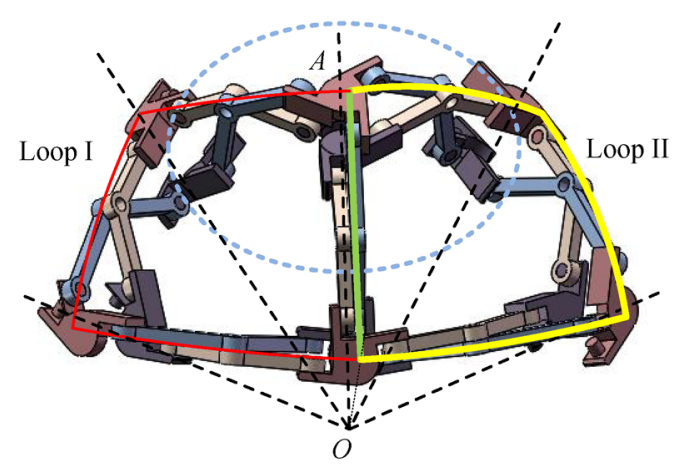

(a)

$$
\left\{\begin{array}{l}
l_{p 2}=l_{q 2}=l_{r 2}, \\
m_{p}=m_{q}=m_{r}, \\
\theta_{p}^{\prime \prime}=\theta_{q}^{\prime \prime}=\theta_{r}^{\prime \prime} \\
\frac{l_{p 2}}{\sin \theta_{p}^{\prime \prime}}=\frac{l_{p 1}}{\sin \theta_{p}^{\prime}}=\frac{l_{q 1}}{\sin \theta_{q}^{\prime}}=\frac{l_{r 1}}{\sin \theta_{r}^{\prime}},
\end{array}\right.
$$

where $l_{j 1}$ and $l_{j 2}$ are the lengths of the links of the $j$ th GAEs II, $m_{j}$ is the ratio of the $j$ th GAEs II, $\theta_{j}^{\prime}$ and $\theta_{j}^{\prime \prime}$ are the angles of the axes of the $j$ th GAEs II, and $j=p, r$, and $q$.

The connection conditions of multiple loops can be inferred from the connection conditions of two loops. All loops have a common deployable center, and adjacent loops share a common deployment axis. Accordingly, we can simplify the connection of multiple loops as one loop connected with another loop. Thus, a Hoberman sphere mechanism based on multiple deployment axes that have a common deployable center needs to satisfy the following constraint conditions: The angles and lengths of links around each deployment axis are equal, the lengths of all the links divided by the sine of the corresponding angles are equal, and ratios $m_{i}$ are all equal.

Next, the steps of constructing a Hoberman sphere mechanism are provided. First, according to the given relationships of the deployment axes and loops, one loop is set as the first loop to determine whether Eq. (28) or (29) is satisfied and whether Eq. (26) has a positive solution.

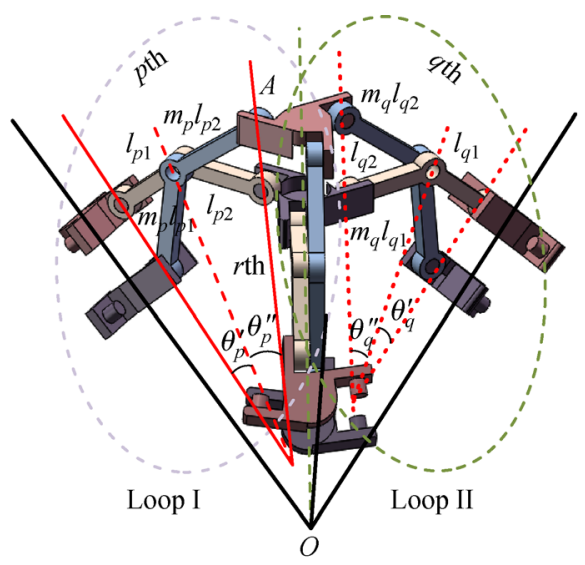

(b)

Fig. 6 Connection for two loops. (a) Two loops; (b) three GAEs II of platform $A$. 
Second, if the first step is satisfied, then the length of a certain link, the corresponding angle, and the ratio $m_{1}$ are given. The first loop can be constructed according to Eq. (24) and the solution of Eq. (26). Third, the next loop that is connected to an axis of the first loop is constructed according to Eqs. (30) and (24) to determine if Eq. (26) has a positive solution. All other loops are similarly designed and connected. Finally, a Hoberman sphere mechanism is constructed, and the corresponding flowchart is shown in Fig. 7.

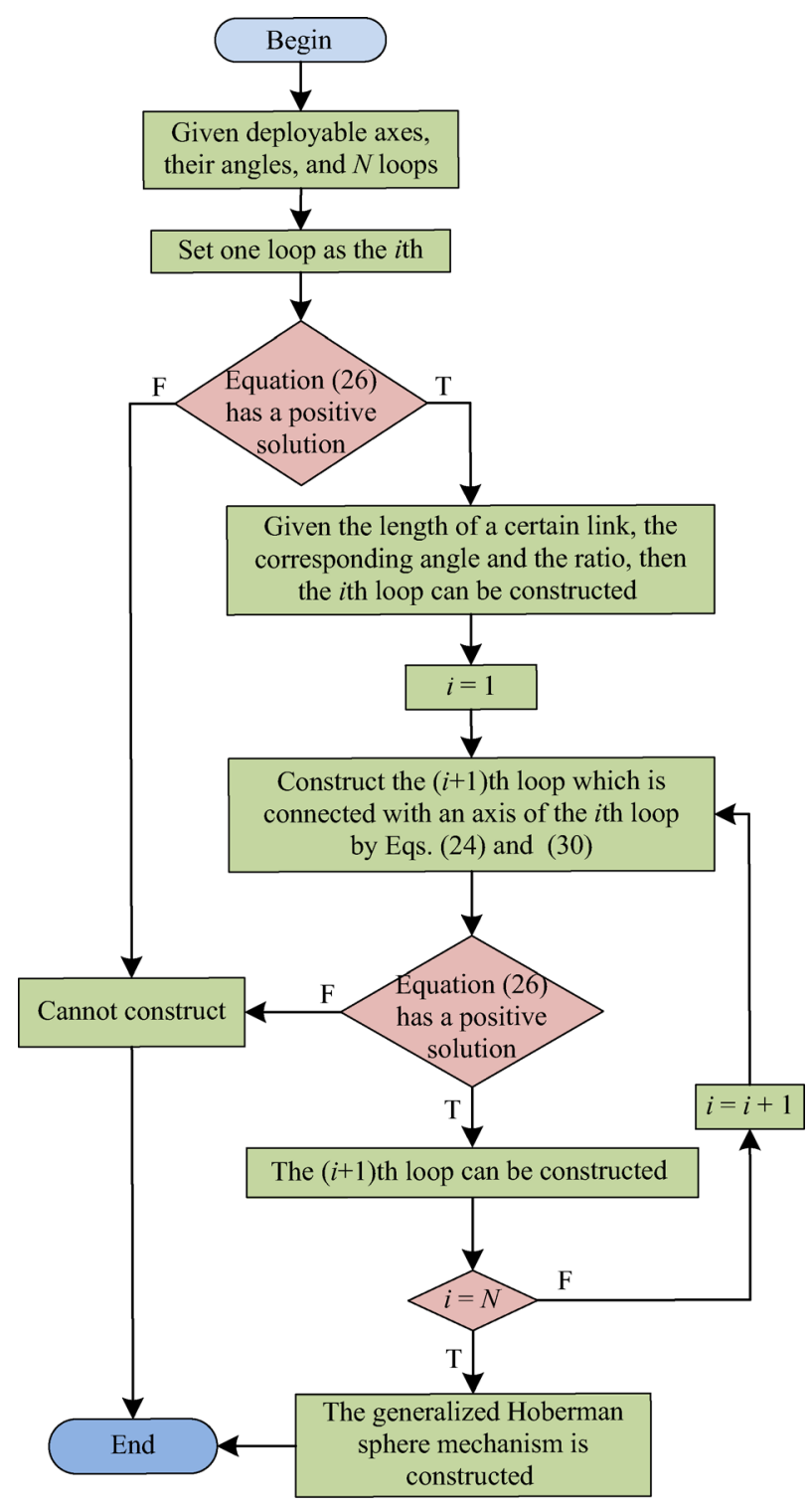

Fig. 7 Flowchart of constructing a Hoberman sphere mechanism.

As illustrated in the flowchart of constructing a generalized Hoberman sphere mechanism, a Hoberman sphere mechanism based on the axes of a regular hexahedron can be constructed only when Eq. (26) has a positive solution. As shown in Fig. 8(a), the deployment axes are the lines connecting the vertexes to the center of a regular polyhedron and the lines connecting the center of the polyhedron to the central point of each side. The center of the regular polyhedron is regarded as the deployable center. Given that the sides of the regular hexahedron are equal, and the angles of the axes are all equal and uniformly denoted by $\theta=35.27^{\circ}$. A loop is considered, and infinite positive solutions can be obtained according to Eq. (26). A positive solution is taken, and the loop is constructed. $m=1.3$ is set, the angles of axes associated with the $i$ th GAEs II are $15.98^{\circ}$ and $19.29^{\circ}$, and the lengths of the links of GAEs II are 50 and $60 \mathrm{~mm}$. The five other loops are designed in the same manner, and all of the six loops are connected by platforms according to Eq. (30). Then, a Hoberman sphere mechanism based on the regular hexahedron is constructed. Its configurations are shown in Figs. 8(b)-8(d).

\section{Generalized Hoberman sphere mechanism with a given arrangement of the deployment axes}

Section 3 indicated that constraint conditions must be satisfied to construct a Hoberman sphere mechanism based on existing and fixed deployment axes. In the current section, the deployment axes are arranged according to the constraints in Eqs. (24), (26), and (30). As shown in Fig. 9(a), six axes are inserted into the six faces of the regular hexahedron, and the angles between the inserted and deployment axes are equal and uniformly denoted by $\zeta=54.74^{\circ}$. GAEs II are then inserted according to Eqs. (24) and (30). The configurations of the Hoberman sphere mechanism based on the fully enclosed regular hexahedron are shown in Figs. 9(b)-9(d). This mechanism possesses more rigidity than the mechanism in Fig. 8. As shown in Fig. 10, a $3 \mathrm{D}$ printed prototype is fabricated to verify the feasibility of the mechanism, and the configurations are shown in Figs. 10(a)-10(c).

Next, a series of axes that satisfy the arithmetic sequence are given. As shown in Fig. 11, the angles of the deployment axes satisfy the arithmetic sequence $\theta_{1}+$ $7 d=\theta_{2}+6 d=\theta_{3}+5 d=\cdots=\theta_{7}+d=\theta_{8} \quad(d$ is a common difference), and the angles satisfy Eqs. (28) and (30). $m=1.3$ is given, and the parameters of the mechanism are obtained as in Table 1 , where $\theta_{i}, \theta_{i}^{\prime}$, and $\theta_{i}^{\prime \prime}$ are the angles of axes associated with the $i$ th GAEs II and $l_{2 i-1}$ and $l_{2 i}$ are the lengths of the links of the $i$ th GAEs II. Therefore, five GAEs II chains that are based on the arithmetic sequence axes are connected by four platforms. The configurations of the Hoberman sphere mechanism based on the arithmetic sequence axes are shown in Figs. 11(b)-11(d).

A series of complicated axes are arranged, as shown in Fig. 12. Two GAEs II loops are inserted into the two series 


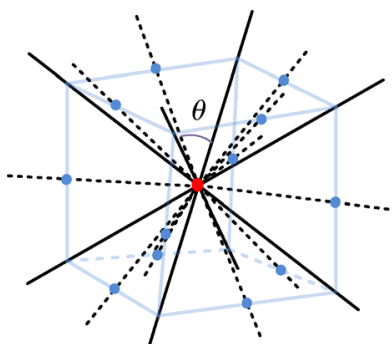

(a)

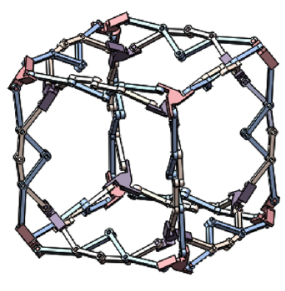

(b)

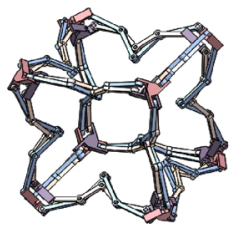

(c)

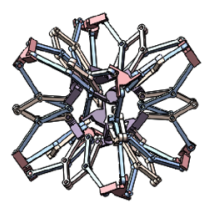

(d)

Fig. 8 Hoberman sphere mechanism based on the regular hexahedron. (a) Deployment axes; (b) configuration I; (c) configuration II; (d) configuration III.

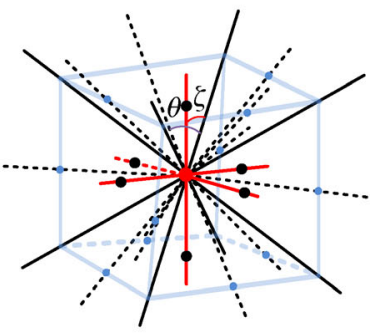

(a)

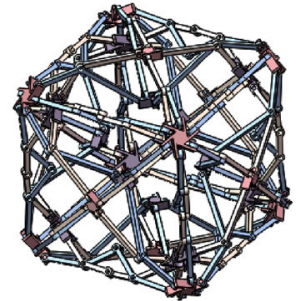

(b)

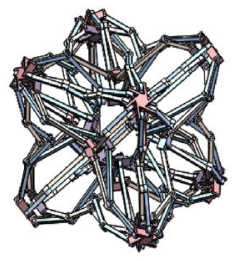

(c)

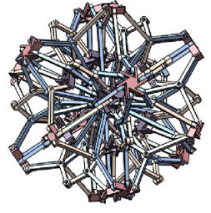

(d)

Fig. 9 Hoberman sphere mechanism based on a fully enclosed regular hexahedron. (a) Insert deployment axes; (b) configuration I; (c) configuration II; (d) configuration III.

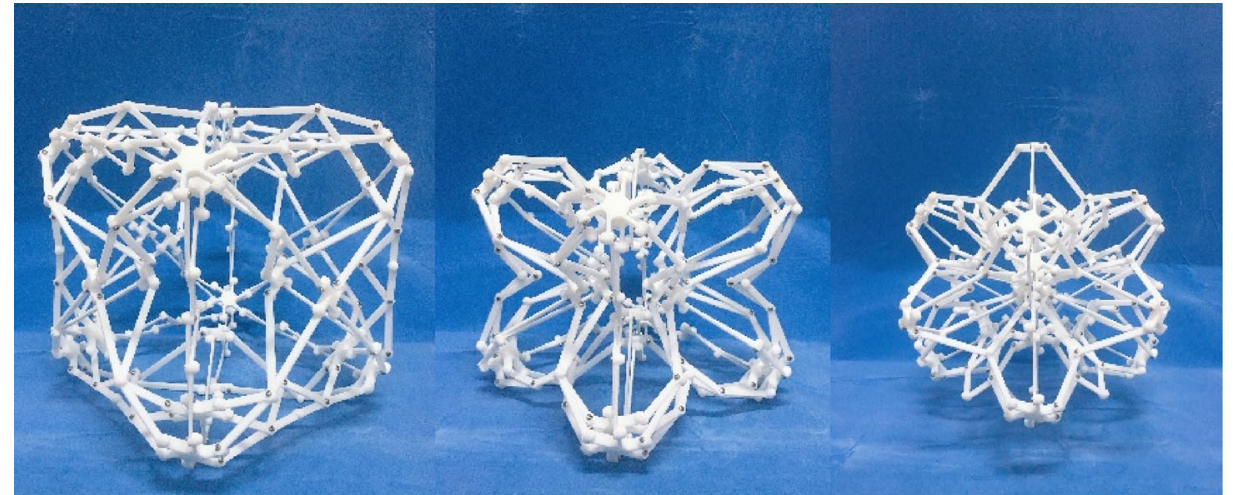

(a) (b) (c)

Fig. 10 3D printed prototype of a Hoberman sphere mechanism based on a fully enclosed regular hexahedron. (a) Configuration I; (b) configuration II; (c) configuration III.

of arithmetic sequence axes separately. The angles of the two loops are of an arithmetic sequence, and the two loops are orthogonal to each other and connected by platforms. According to Eq. (28), $m=1.3$ is given, and the lengths of the links and angles are as shown in Table 1. Then, as shown in Fig. 12(a), 20 axes are inserted to connect the two orthogonal loops, which belong to four types. The angles and lengths of the links are determined by Eqs. (24) and (30), as shown in Table 2 , where $\zeta_{j}, \zeta_{j}^{\prime}$, and $\zeta_{j}^{\prime \prime}$ are the angles associated with the $j$ th GAEs II and $L_{2 j-1}$ and $L_{2 j}$ are the lengths of the $j$ th GAEs II. A Hoberman sphere mechanism based on the spiral-like axes is then constructed (Fig. 13), and 23 axes are arranged cylindrically, as shown in Fig. 13(a). The height of the cylinder is 300 $\mathrm{mm}$, the diameter is $300 \mathrm{~mm}$, and the cylinder is cut evenly into six parts at a distance of $50 \mathrm{~mm}$. The center point of the cylinder is the deployable center. Three uniform points are obtained on the upper bottom surface of the cylinder and rotated by $60^{\circ}$ on the second surface until they are rotated to the lower bottom surface. The lines connecting 


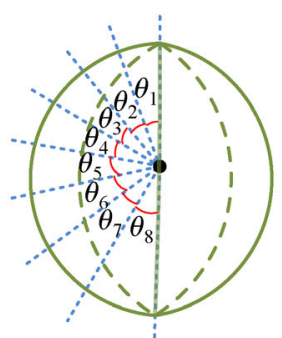

(a)

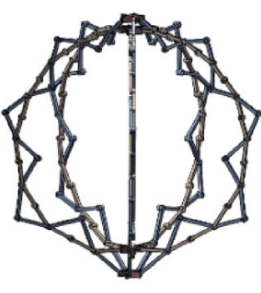

(b)

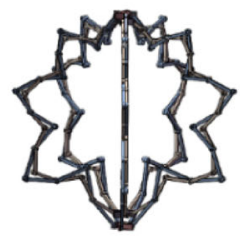

(c)

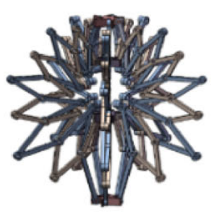

(d)

Fig. 11 Hoberman sphere mechanism based on arithmetic sequence axes. (a) Deployment axes; (b) configuration I; (c) configuration II; (d) configuration III.

Table 1 Parameters of the Hoberman sphere mechanism based on arithmetic sequence axes

\begin{tabular}{|c|c|c|c|c|c|}
\hline$i$ & $\theta_{i} /\left(^{\circ}\right)$ & $\theta_{i}^{\prime} /\left(^{\circ}\right)$ & $\theta_{i}^{\prime \prime} /\left(^{\circ}\right)$ & $l_{2 i-1} / \mathrm{mm}$ & $l_{2 i} / \mathrm{mm}$ \\
\hline 1 & 15.5 & 7.25 & 8.25 & 37.86 & 43.05 \\
\hline 2 & 17.5 & 8.25 & 9.25 & 43.05 & 48.22 \\
\hline 3 & 19.5 & 9.25 & 10.25 & 48.22 & 53.38 \\
\hline 4 & 21.5 & 10.25 & 11.25 & 53.38 & 58.53 \\
\hline 5 & 23.5 & 11.25 & 12.25 & 58.53 & 63.65 \\
\hline 6 & 25.5 & 12.25 & 13.25 & 63.65 & 68.76 \\
\hline 7 & 27.5 & 13.25 & 14.25 & 68.76 & 73.85 \\
\hline 8 & 29.5 & 14.25 & 15.25 & 73.85 & 78.91 \\
\hline
\end{tabular}

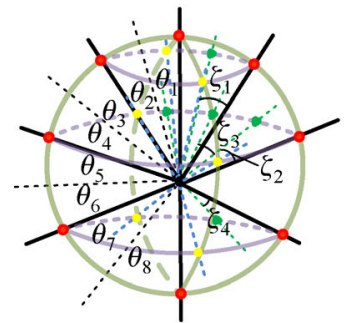

(a)

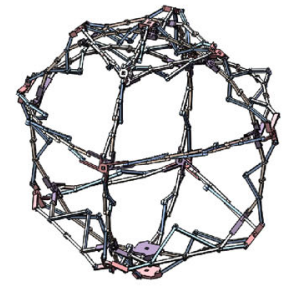

(b)

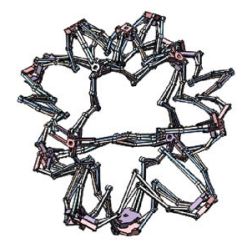

(c)

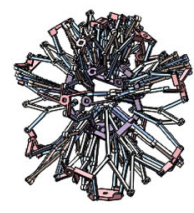

(d)

Fig. 12 Hoberman sphere mechanism based on orthonormal arithmetic sequence axes. (a) Deployment axes; (b) configuration I; (c) configuration II; (d) configuration III.

Table 2 Parameters of inserted axes

\begin{tabular}{|c|c|c|c|c|c|}
\hline$j$ & $\zeta_{j} /\left(^{\circ}\right)$ & $\zeta_{j}^{\prime} /\left(^{\circ}\right)$ & $\zeta_{j}^{\prime \prime} /\left(^{\circ}\right)$ & $L_{2 j-1} / \mathrm{mm}$ & $L_{2 j} / \mathrm{mm}$ \\
\hline 1 & 22.65 & 9.25 & 13.40 & 48.22 & 69.53 \\
\hline 2 & 22.50 & 11.25 & 11.25 & 58.53 & 58.53 \\
\hline 3 & 20.32 & 11.25 & 9.07 & 58.53 & 47.30 \\
\hline 4 & 36.37 & 13.25 & 23.12 & 68.76 & 117.81 \\
\hline
\end{tabular}

these points to the deployable center are set as the deployment axes, which satisfy the relationships in Eqs. (24) and (30). Then, the platforms and GAEs II are inserted, and $m=1.3$ is given. The lengths of the links and the other parameters are shown in Table 3.

\section{Conclusions}

The connections of two GAEs II with three deployment axes are analyzed in three cases, and the constraint conditions for one loop with $n$ GAEs II are obtained for 


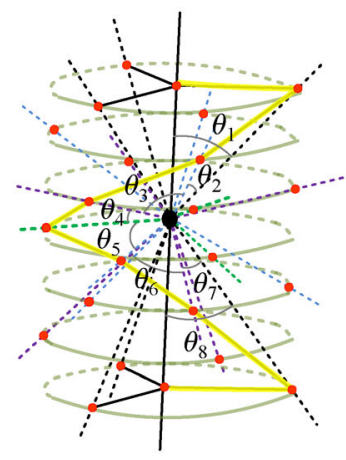

(a)

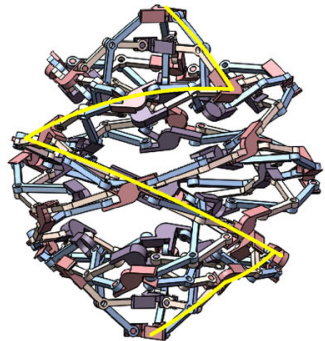

(b)

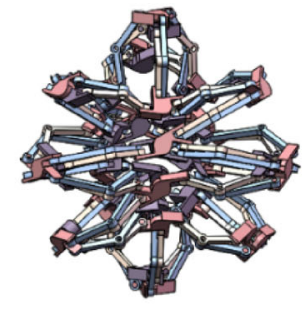

(c)

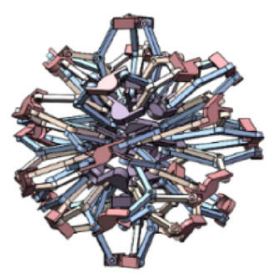

(d)

Fig. 13 Hoberman sphere mechanism based on spiral-like axes. (a) Deployment axes; (b) configuration I; (c) configuration II; (d) configuration III.

Table 3 Parameters of the Hoberman sphere mechanism based on spiral-like axes

\begin{tabular}{|c|c|c|c|c|c|}
\hline$i$ & $\theta_{i} /\left(^{\circ}\right)$ & $\theta_{i}^{\prime} /\left(^{\circ}\right)$ & $\theta_{i}^{\prime \prime} /\left(^{\circ}\right)$ & $l_{2 i-1} / \mathrm{mm}$ & $l_{2 i} / \mathrm{mm}$ \\
\hline 1 & 45.00 & 22.50 & 22.50 & 45.00 & 45.00 \\
\hline 2 & 46.65 & 22.50 & 24.15 & 45.00 & 48.12 \\
\hline 3 & 55.24 & 24.15 & 31.09 & 48.12 & 60.72 \\
\hline 4 & 61.68 & 31.09 & 30.59 & 60.72 & 59.85 \\
\hline 5 & 61.68 & 30.59 & 31.09 & 59.85 & 60.72 \\
\hline 6 & 55.24 & 31.09 & 24.15 & 60.72 & 48.12 \\
\hline 7 & 46.65 & 24.15 & 22.50 & 48.12 & 45.00 \\
\hline 8 & 45.00 & 22.50 & 22.50 & 45.00 & 45.00 \\
\hline
\end{tabular}

the first time based on the common center and $n$ deployment axes. A highly general approach is proposed and verified by examples to construct a generalized Hoberman sphere mechanism based on the GAEs II loops and deployment axes. A series of novel generalized Hoberman sphere mechanisms are constructed by designing the parameters of GAEs II loops and arranging the deployment axes, such as arithmetic sequence, orthonormal arithmetic sequence, and spiral-like axes. The obtained mechanisms can expand the application scope of traditional Hoberman sphere mechanisms.

Acknowledgements This work was supported by the National Natural Science Foundation of China (Grant No. 51905015) and China Postdoctoral Science Foundation (Grant No. 2018M631300).

Open Access This article is licensed under a Creative Commons Attribution 4.0 International License, which permits use, sharing, adaptation, distribution, and reproduction in any medium or format, as long as you give appropriate credit to the original author(s) and the source, provide a link to the Creative Commons license, and indicate if changes were made.

The images or other third-party materials in this article are included in the article's Creative Commons license, unless indicated otherwise in a credit line to the material. If a material is not included in the article's Creative Commons license and your intended use is not permitted by statutory regulation or exceeds the permitted use, you will need to obtain permission directly from the copyright holder.

To view a copy of this license, visit http://creativecommons.org/licenses/ by $/ 4.0 \%$.

\section{References}

1. Piñero E P. Project for a mobile theatre. Architectural Design, 1961, 12(1): 154-155

2. Zeigler T R. US Patent, 4290244, 1981-9-22

3. Escrig F. Expandable space structures. International Journal of Space Structures, 1985, 1(2): 79-91

4. Escrig F, Valcarcel J P. Geometry of expandable space structures. International Journal of Space Structures, 1993, 8(1-2): 71-84

5. You Z. Deployable structure of curved profile for space antennas. Journal of Aerospace Engineering, 2000, 13(4): 139-143

6. Hoberman C. US Patent, 4942700, 1990-7-24

7. Hoberman C. US Patent, 5024031, 1991-6-18

8. You Z, Pellegrino S. Foldable bar structures. International Journal of Solids and Structures, 1997, 34(15): 1825-1847

9. Patel J, Ananthasuresh G K. A kinematic theory for radially foldable planar linkages. International Journal of Solids and Structures, 2007, 44(18-19): 6279-6298

10. Cai J, Xu Y, Feng J. Kinematic analysis of Hoberman's linkages with the screw theory. Mechanism and Machine Theory, 2013, 63(63): 28-34

11. Cai J, Deng X, Feng J, et al. Mobility analysis of generalized angulated scissor-like elements with the reciprocal screw theory. Mechanism and Machine Theory, 2014, 82: 256-265

12. Chen Y, Fan L, Feng J. Kinematic of symmetric deployable scissorhinge structures with integral mechanism mode. Computers \& 
Structures, 2017, 191: 140-152

13. Bai G, Liao Q, Li D, et al. Synthesis of scaling mechanisms for geometric figures with angulated-straight elements. Proceedings of the Institution of Mechanical Engineers. Part C, Journal of Mechanical Engineering Science, 2013, 227(12): 27952809

14. Wohlhart K. Cyclic polyhedra and linkages derived therefrom. Mechanism and Machine Theory, 2017, 108: 142-159

15. Wohlhart K. Equally circumscribed cyclic polyhedra generalize Platonic solids. Mechanism and Machine Theory, 2019, 133: 150163

16. Wei G, Dai J S. Overconstrained mechanisms with radially reciprocating motion. In: Lenarcic J, Stanisic M, eds. Advances in Robot Kinematics: Motion in Man and Machine. Dordrecht: Springer, 2010, 293-300

17. Li R, Yao Y A, Kong X. A class of reconfigurable deployable platonic mechanisms. Mechanism and Machine Theory, 2016, 105: 409-427

18. Li R, Yao Y A, Ding X. A family of reconfigurable deployable polyhedral mechanisms based on semiregular and Johnson polyhedral. Mechanism and Machine Theory, 2018, 126: 344-358

19. Li R, Sun X, Chen Y, et al. Design and analysis of reconfigurable deployable polyhedral mechanisms with straight elements. Journal of Mechanisms and Robotics, 2019, 11(4): 044502

20. Kiper G, Söylemez E, Kişisel A U Ö. Polyhedral linkages synthesized using Cardan Motion along radial axes. In: Proceedings of the 12th IFToMM World Congress. Besançon, 2007, 17-21

21. Shieh W B. Design of the deployable mechanisms based on the Cardanic motion of planar four-bar linkage. In: Proceedings of ASME 2014 International Design Engineering Technical Conferences and Computers and Information in Engineering Conference. Buffalo: ASME, 2014, DETC2014-34838 V05AT08A061

22. Huang H, Li B, Zhu J, et al. A new family of Bricard-derived deployable mechanisms. Journal of Mechanisms and Robotics, 2016, 8(3): 034503

23. Huang H, Li B, Zhang T, et al. Design of large single-mobility surface-deployable mechanism using irregularly shaped triangular prismoid modules. Journal of Mechanical Design, 2019, 141(1): 012301

24. Qi X, Huang H, Miao Z, et al. Design and mobility analysis of large deployable mechanisms based on plane-symmetric Bricard linkage. Journal of Mechanical Design, 2017, 139(2): 022302

25. Wang J, Kong X. Deployable polyhedron mechanisms constructed by connecting spatial single-loop linkages of different types and/or in different sizes using S joints. Mechanism and Machine Theory, 2018, 124: 211-225

26. St-Onge D, Gosselin C. Synthesis and design of a one degree-offreedom planar deployable mechanism with a large expansion ratio. Journal of Mechanisms and Robotics, 2016, 8(2): 021025 